\title{
O efeito da temperatura de queima da camada de opaco na resistência flexural de uma cerâmica vítrea submetida à cocção sobre uma liga de cobalto-cromo
}

\section{Opaque layer firing temperature effect on the flexural strength of glass ceramic fused to cobalt-chromium alloy}

\section{Luis Gustavo Oliveira de VASCONCELLOS}

Doutorando - Departamento de Materiais Odontológicos e Prótese - Programa de Pós-Graduação em Odontologia Restauradora - Faculdade de Odontologia de São José dos Campos - UNESP - São José dos Campos - SP - Brasil.

\author{
Lucas Villaça ZOGHEIB \\ Professor Doutor - Disciplina de Prótese Dentária e Clínica Integrada - Faculdade de Odontologia, Universidade de Passo \\ Fundo - UPF - Passo Fundo - RS - Brasil.
}

\section{Rodrigo Othávio de Assunção e SOUZA}

Professor Adjunto - Disciplina de Prótese Fixa - Departamento de Odontologia Restauradora, Universidade Federal da Paraíba - UFPB - João Pessoa - PB - Brasil.

\section{Lafayette NOGUEIRA JUNIOR}

Professor Adjunto - Departamento de Materiais Odontológicos e Prótese - Programa de Pós-Graduação em Odontologia Restauradora - Faculdade de Odontologia de São José dos Campos - UNESP - São José dos Campos - SP - Brasil.

\section{Marco Antônio BOTTINO}

Professor Titular - Departamento de Materiais Odontológicos e Prótese - Faculdade de Odontologia de São José dos Campos - UNESP - São José dos Campos - SP - Brasil.

\begin{abstract}
Resumo
Este estudo avaliou a influência da temperatura de queima da camada de opaco na resistência de união entre uma liga de cobalto-cromo e uma cerâmica, após serem submetidos ao ensaio de flexão de três pontos. Considera-se a hipótese: o aumento da temperatura de queima do opaco melhora a resistência flexural da cerâmica aplicada sobre o metal. Barras metálicas $(25 \times 3 \times 0,5 \mathrm{~mm})(\mathrm{n}=30)$ foram fundidas em $\mathrm{Co}-\mathrm{Cr}$ e as áreas centrais das barras $(8 \times 3 \mathrm{~mm})$ foram jateadas com partículas de óxido de alumínio de $150 \mu \mathrm{m}$ e divididas em 3 grupos de acordo com a temperatura de queima da camada de opaco ( $\mathrm{n}=10)$ : $\mathrm{G} 1$ (grupo controle) $-900^{\circ} \mathrm{C} ; \mathrm{G} 2-950^{\circ} \mathrm{C}$ e G3 $-1000^{\circ} \mathrm{C}$. As cerâmicas opaca e de corpo (Vita Omega 900) foram queimadas sobre a área central. Os espécimes foram mantidos em água destilada à $37^{\circ} \mathrm{C}$ durante 24 horas e a seguir foram submetidos ao teste de flexão de três pontos. Após o teste, os tipos de falhas foram classificados. Os dados obtidos foram analisados estatisticamente (Kruskal-Wallis 1 e teste de Dunn , $\mu=0,05$ ). A diferença dos valores médios de resistência flexural foi estatisticamente significante $(\mathrm{p}=0,004)$, sendo que os grupos G2- $19,84 \pm 4,24 \mathrm{~N}$ e G3- $22,43 \pm 5,94 \mathrm{~N}$ exibiram valores de resistência flexural maiores que o grupo G1- $12,525 \pm 1,08 \mathrm{~N}$. Concluíu-se que o aumento da temperatura de queima da camada de opaco melhorou significantemente os valores de resistência flexural. O modo de falha predominante foi adesiva na interface metal/cerâmica para o grupo G1 e exclusivamente coesiva na zona interfacial camada de opaco/Co-Cr com presença de cerâmica na superfície dos grupos G2 e G3.
\end{abstract}

\section{UNITERMOS}

Materiais dentários; ligas metalocerâmicas; ligas de cromo; cerâmica.

\section{INTRODUÇÃO}

O alto custo das ligas metálicas nobres tem levado ao desenvolvimento e ao aumento do uso clínico das ligas de metais básicos para confecção de infra-estru- turas (IE) de próteses parciais fixas e unitárias ${ }^{2,21,28}$. Dentre os metais básicos, as ligas de níquel-cromo e cobalto-cromo são as mais comumente usadas ${ }^{19,28}$. As ligas de níquel-cromo com berílio podem apresentar 
os seguintes problemas: a) toxicidade relacionada ao berílio; b) potencial alérgico referente ao níquel ${ }^{3,19}$. Estes fatores determinam o emprego mais intenso das ligas de cobalto-cromo devido a sua compatibilidade biológica ${ }^{3}$. As ligas não-nobres apresentam excelentes propriedades mecânicas ${ }^{2,21,28}$, tais como resistência à deformação permanente e alto módulo de elasticidade, que lhes conferem a vantagem de obter infra-estruturas com pequena espessura e com a rigidez requerida para próteses parciais fixas extensas ${ }^{19,28}$.

Falhas na união metal/cerâmica são comuns após a cimentação de próteses parciais fixas com IE em ligas alternativas. Provavelmente estas falhas ocorrem durante a fase laboratorial da confecção da restauração e se manifestam clinicamente com as forças geradas durante ou após o procedimento de cimentação ${ }^{16}$. Um dos principais fatores responsáveis por estas falhas é a dificuldade de se controlar a formação da camada de óxido na superfície do metal, em temperaturas elevadas, afetando negativamente a união entre metal e a cerâmica ${ }^{6,8,22}$.

Pequenas variações nos procedimentos laboratoriais podem causar diferença significante na resistência de união entre metal e cerâmica, por isso a obediência a detalhes durante a confecção de prótese parciais fixas no laboratório são essenciais para o sucesso da restauração ${ }^{28}$. Modificações nos procedimentos laboratoriais são sugeridas por alguns autores, visando aumentar a resistência de união entre os materiais, tais como: uso de agentes intermediários de união ${ }^{13,23}$, diferentes temperaturas de queima da cerâmica ${ }^{5}$, alteração na taxa de resfriamento da cerâmica ${ }^{16}$, utilização de diferentes ambientes para queima da cerâmica ${ }^{10,28}$, aumento do número de queimas da cerâmica ${ }^{4,24}$, modificação no tratamento do metal ${ }^{7,15}$, uso da camada de opaco ${ }^{20}$ e aumento da temperatura de queima da camada de opaco ${ }^{8,17,28}$

$\mathrm{O}$ objetivo deste estudo foi avaliar in vitro a influência de três diferentes temperaturas de queima da camada de opaco na união de um metal a uma cerâmica, quando submetidos ao ensaio de flexão de três pontos. A hipótese levada em consideração foi: o aumento da temperatura de queima do opaco melhora a resistência flexural da cerâmica ao metal.

\section{Material e Método}

\section{Fabricação das barras metálicas}

Tiras plásticas $(27 \times 3 \times 0,5 \mathrm{~mm})$ foram utilizadas para a fabricação das barras metálicas. Sprues de cera (Horus, Herpo Produtos Dentários Ltda, São Paulo, Brasil) foram unidos perpendicularmente a uma das extremidades das tiras plásticas e conectados a um bastão de cera central com $5 \mathrm{~mm}$ de diâmetro (Horus, Herpo Produtos Dentários Ltd, São Paulo, Brasil). O conjunto foi montado em um anel de silicone e preenchido com material de revestimento (Bellavest ${ }^{\mathbb{B}} \mathrm{T}$, Bego, Bremen, Alemanha). Após a presa do material, o anel de silicone foi separado do molde de revestimento. Em liga de cobalto-cromo (Wirobond ${ }^{\circledR} \mathrm{C}$, Bego, Alemanha) foram obtidas trinta barras metálicas após a fusão em equipamento para fundição por indução (Fornax GEU, Bego, Bremen, Alemanha).

Após os procedimentos de desinclusão, com auxílio de um disco de carborundum em baixa velocidade, os condutos de alimentação foram cortados, as irregularidades removidas e a extremidade que estava ligada ao conduto de alimentação, recortada. Finalmente, as barras metálicas apresentaram as seguintes dimensões de $25 \times 3 \times 0,5 \mathrm{~mm}$, seguindo a norma ISO 969318. As dimensões foram aferidas por meio de um paquímetro digital com resolução de $0,01 \mathrm{~mm}$ (modelo Starrett 727, Starrett, Itu, Brasil).

Um dos lados das barras metálicas foi selecionado aleatoriamente e usinado com pedra branca de óxido de alumínio de formato cilíndrico (Shofu, Menlo Park, EUA). Em seguida, o lado usinado foi jateado com pó de óxido de alumínio de 150um (Korox, BEGO, Bremen, Alemanha), sob pressão de 50lb com inclinação de $45^{\circ}$ e distância de $2 \mathrm{~cm}$ por um período de 10 segundos. Posteriormente, foi realizada a limpeza com banho sônico (Vitasonic II, Vita Zahnfabrick, Bad Säckingen, Alemanha) em álcool isopropílico por um período de dez minutos. As barras metálicas foram mantidas em bancada sobre papel absorvente para total secagem.

\section{Aplicação da cerâmica}

Para a aplicação da cerâmica, foi desenvolvido um dispositivo metálico, o qual possuía duas placas horizontais, uma superior e outra inferior, unidas por uma das extremidades. A placa horizontal inferior apresentava uma depressão longitudinal na extremidade livre, com dimensões de $16 \times 3 \times 1,5 \mathrm{~mm}$ e uma depressão transversal, a uma distância de $8 \mathrm{~mm}$ da parte mais externa do dispositivo, que permitia posicionar o arame de travamento, fixando a barra metálica. A área que se estendia do arame de travamento até a parte mais externa do dispositivo, apresentava dimensões de $8 \times 3 \mathrm{~mm}^{18}$. Além disso, esta depressão longitudinal possuía profundidade de $1,5 \mathrm{~mm}$, permitindo $1,0 \mathrm{~mm}$ de espaço para aplicação da cerâmica e $0,5 \mathrm{~mm}$ relativo à espessura da barra (Figura 1). 

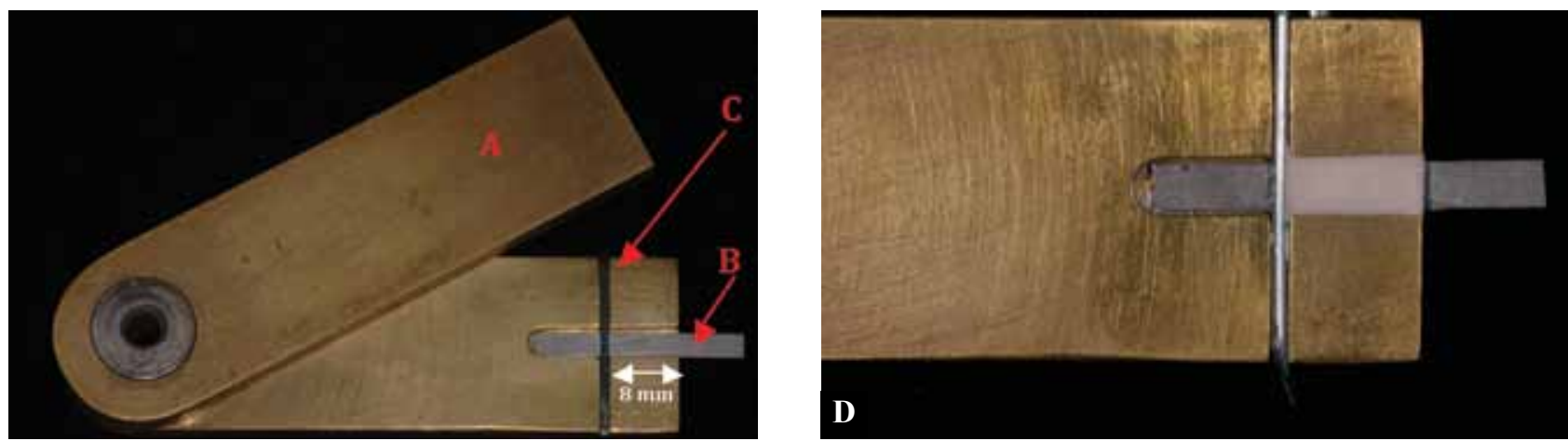

Figura 1 - a) Dispositivo metálico utilizado para aplicação da cerâmica, dimensões de acordo com ISO 9396, b) barra metálica posicionada no dispositivo, c) arame de travamento fixando a barra metálica, antes aplicação da camada de opaco, d) após a aplicação da camada de cerâmica.

Com auxílio de um pincel, uma fina camada de opaco (Wash Opaque WO 9000, Vita Zahnfabrick, Bad Säckingen, Alemanha) foi aplicada na superfície delimitada pelo dispositivo. O ciclo de queima do opaco foi conduzido em forno elétrico (Vacumat 40, Vita Zahnfabrick, Bad Säckingen, Alemanha), variando a temperatura de queima da camada de opaco (Quadro 1).

Em seguida, uma camada de cerâmica opaca (1M1 9301, Vita Omega 900, Vita Zahnfabrick, Bad
Säckingen, Alemanha) e duas camadas de cerâmica de dentina (4L1.5 9377, Vita Omega 900, Vita Zahnfabrick, Bad Säckingen, Alemanha) foram aplicadas e levadas ao forno elétrico para queima, de acordo com as recomendações do fabricante (Quadro 2). A segunda camada de cerâmica de dentina foi aplicada visando corrigir defeitos e compensar a contração de queima da cerâmica. A amostra final apresentou dimensões de cerâmica de $8 \times 3 \times 1 \mathrm{~mm}$.

Quadro 1 - Procedimentos de queima da camada de opaco Wash Opaque para todos os grupos.

\begin{tabular}{lccccc}
\hline Cerâmicas & $\begin{array}{c}\text { Pré-secagem } \\
(\mathbf{m i n})\end{array}$ & $\begin{array}{c}\text { Temperatura inicial } \\
\left({ }^{\circ} \mathbf{C}\right)\end{array}$ & $\begin{array}{c}\text { Taxa de } \\
\text { aumento } \\
\left({ }^{\circ} \mathbf{C} / \mathbf{m i n}\right)\end{array}$ & $\begin{array}{c}\text { Temperatura } \\
\text { Final } \\
\left({ }^{\circ} \mathbf{C}\right)\end{array}$ & $\begin{array}{c}\text { Tempo de queima } \\
(\mathbf{m i n})\end{array}$ \\
\hline Vita Omega $\mathbf{9 0 0}$ & & & & & \\
Gr1 (controle) & 4 & 600 & 75 & 900 & 1 \\
Gr2 & 4 & 600 & 88 & 950 & 1 \\
Gr3 & 4 & 600 & 100 & 1000 & 1 \\
\hline
\end{tabular}

Quadro 2 - Procedimentos de queima da cerâmica testada.

\begin{tabular}{lccccc}
\hline Cerâmicas & $\begin{array}{c}\text { Pré-secagem } \\
(\mathbf{m i n})\end{array}$ & $\begin{array}{c}\text { Temperatura } \\
\text { inicial } \\
\left({ }^{\circ} \mathrm{C}\right)\end{array}$ & $\begin{array}{c}\text { Taxa de } \\
\text { aumento } \\
\left({ }^{\circ} \mathbf{C} / \mathbf{m i n}\right)\end{array}$ & $\begin{array}{c}\text { Temperatura } \\
\text { Final } \\
\left({ }^{\circ} \mathrm{C}\right)\end{array}$ & $\begin{array}{c}\text { Tempo de } \\
\text { queima } \\
(\mathbf{m i n})\end{array}$ \\
\hline Vita Omega 900 & & & & & \\
Dentina opaca & 4 & 600 & 75 & 900 & 1 \\
Primeira camada de dentina & 4 & 600 & 75 & 900 & 1 \\
Segunda camada de dentina & 6 & 600 & 50 & 900 & 1 \\
\hline
\end{tabular}




\section{Análise da interface metal cerâmica (SEM / EDS)}

Adicionalmente aos grupos experimentais, foram confeccionados dois espécimes de cada grupo, os quais não foram submetidos ao teste de flexão. Os espécimes foram embebidos em resina acrílica autopolimerizável, seccionados longitudinalmente, polidos com auxílio de lixas d'água com granulação de 220 a 1200 e pasta de diamante $(6,3$, and $0,25 \mu \mathrm{m})$ sob irrigação de água constante (POLI PAN-2/Panambra, Sao Paulo, SP, Brasil).

A análise da morfologia da interface metal/cerâmica foi realizada em cada grupo por meio microscopia eletrônica de varredura (MEV) (LEO 435 VPI/LEOZeiss, Tokyo, Japão). Os dados foram coletados ao longo da interface metal/cerâmica das amostras e sob aumento de 150x, uma área significativa da interface foi selecionada e registrada.

\section{Teste de flexão de três pontos}

Os corpos de prova foram submetidos ao ensaio mecânico de flexão de três pontos, em máquina de ensaio universal EMIC modelo DL-1000 (EMIC Curitiba - Brasil), com célula de carga de $10 \mathrm{Kg}$ e velocidade de $1,5 \mathrm{~mm} / \mathrm{min}$. Os corpos de prova foram apoiados em dispositivo de sustentação com apoios de $3 \mathrm{~mm}$ de diâmetro e distância entre os apoios de $20 \mathrm{~mm}$. A face revestida de cerâmica foi posicionada para baixo e o carregamento foi realizado na porção central do corpo de prova, por meio de haste conforme ilustrado na Figura 2.

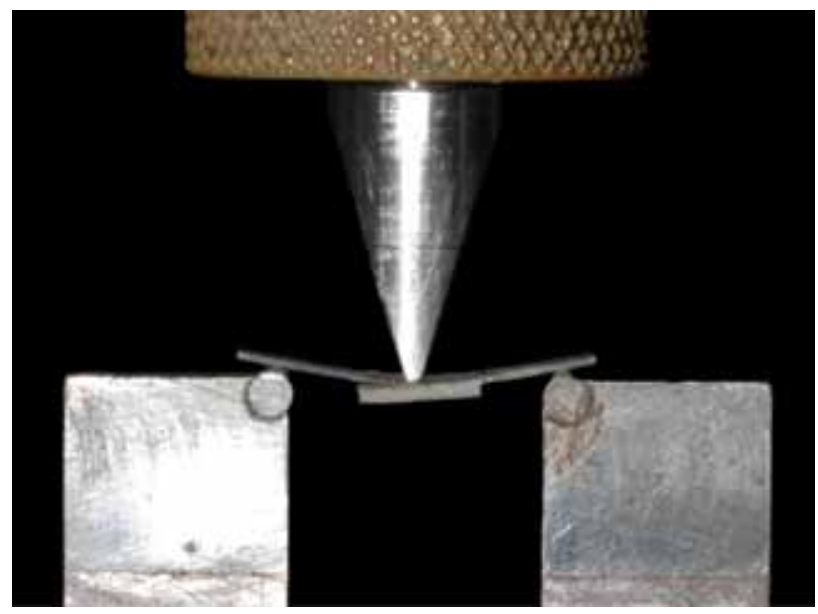

Figura 2 - Aplicação da carga sobre o metal até a ruptura da cerâmica da superfície do metal.
Os dados obtidos em Kgf foram transformados para unidade Newton $(\mathrm{N})$, por meio da seguinte fórmula:

$$
\mathrm{N}=\mathrm{F}(\mathrm{Kgf}) \times 9,8 \mathrm{~m} / \mathrm{s}
$$

\section{Análise do modo de fratura}

Após o ensaio mecânico de flexão, as superfícies de todos os corpos de prova foram analisadas em lupa estereomicroscópica (Stemi 2000-C; Carl Zeiss, Gottingen, Alemanha) com aumento de $30 \mathrm{x}$ e fotografadas por meio de uma câmera digital (Cybershot, model DSC S85, Sony, Tóquio, Japão) acoplada a lupa estereomicroscópica, visando classificar o modo de falha.

Os modos de falhas foram classificados como: a) adesiva ao longo da interface cerâmica opaca e zona de interação; b) dentro da zona de interação; c) coesiva ao longo da interface metal e zona de interação.

\section{Análise estatística}

Os dados obtidos no ensaio de flexão de três pontos foram submetidos à analise estatística por meio dos programas Minitab (Minitab, version 14.12,2004) e Statistix (Analytical Software Inc., Versão 8.0, 2003).

As diferentes temperaturas de queima da camada de opaco (G1, G2 e G3) foram consideradas variáveis independentes. A força $(\mathrm{N})$ necessária para causar a fratura na interface metal-cerâmica foi considerada a variável dependente. Os resultados foram submetidos ao teste estatístico não paramétrico Kruskal-Wallis e ao teste de Dunn (5\%).

\section{Resultados}

Os valores médios de resistência flexural $(\mathrm{N})$ e os desvios-padrões $( \pm \mathrm{DP})$ para as condições experimentais dos grupos foram: $\mathrm{G} 1=12,52 \pm 1,07 ; \mathrm{G} 2=$ $19,84 \pm 4,24$ e G3=22,43 $\pm 5,94$; e estão representados na Figura 3.

Para a melhor interpretação dos dados sobre a resistência flexural, foram utilizados os testes estatísticos de Kruskal-Wallis e de Dunn. O teste de KruskalWallis indicou que a distribuição dos valores não é a mesma entre os três grupos $(\mathrm{kw}=15,811 ; \mathrm{gl}=2 ; \mathrm{p}=$ $0,0004<0,05)$. O teste de Dunn ( $5 \%$ ) indicou que o G1 diferiu dos grupos G2 e G3 que, entre si, não diferem estatisticamente (Tabela 1). 


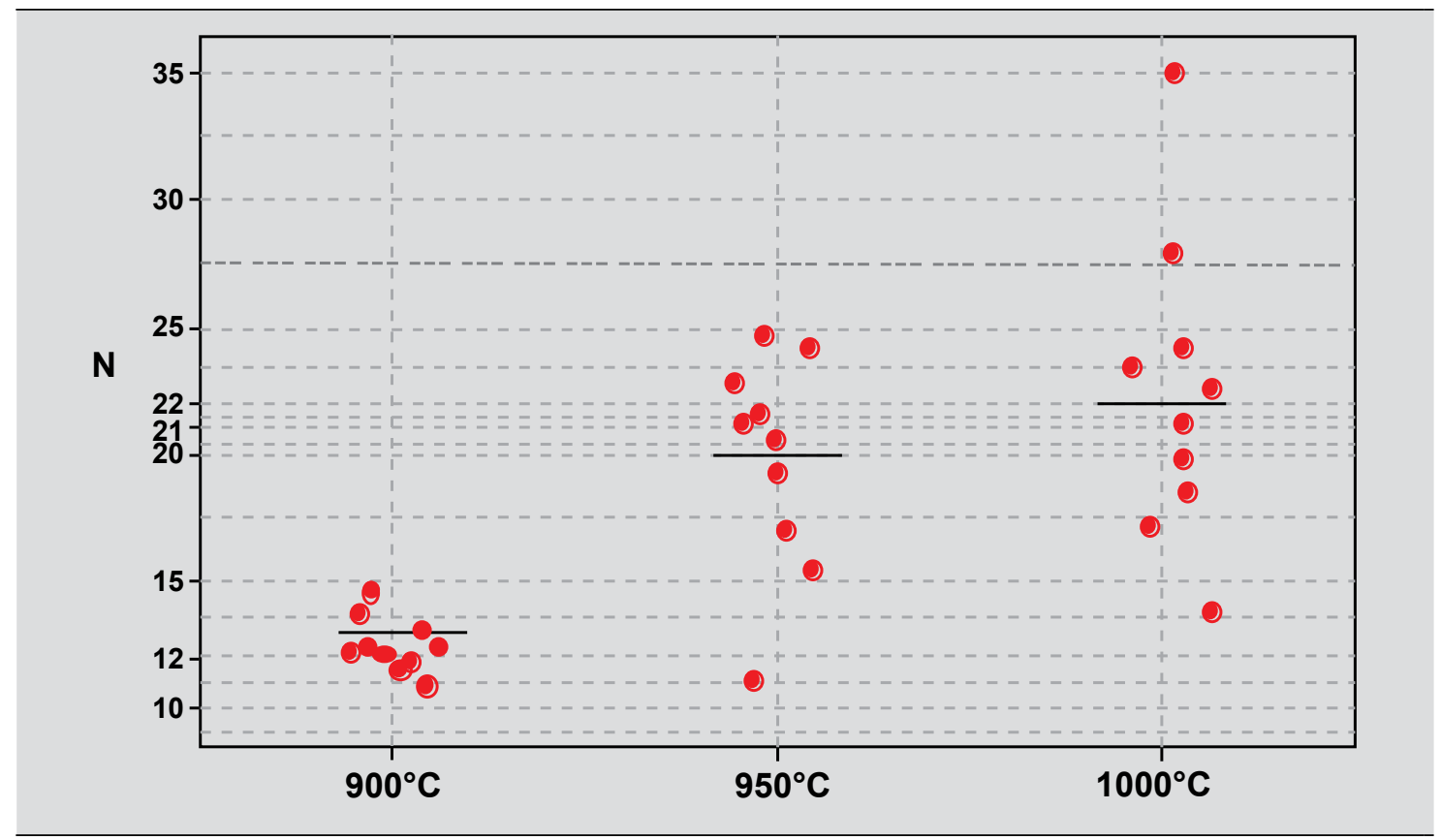

Figura 3 - Médias dos valores de resistência flexural (N) de acordo com a temperatura de queima da camada de opaco.

Tabela 1 - Resultado da comparação das medianas das três condições experimentais, após a aplicação do teste de Dunn (5\%).

\begin{tabular}{lcc}
\hline $\begin{array}{c}\text { Grupos } \\
\text { experimentais }\left({ }^{\circ} \mathrm{C}\right)\end{array}$ & Mediana & $\begin{array}{c}\text { Grupos } \\
\text { homogêneos* }\end{array}$ \\
\hline $\mathrm{G} 31000^{\circ} \mathrm{C}$ & 22,0 & $\mathrm{~A}$ \\
$\mathrm{G} 2950^{\circ} \mathrm{C}$ & 20,92 & $\mathrm{~A}$ \\
$\mathrm{G} 1900^{\circ} \mathrm{C}$ & 12,4 & $\mathrm{~B}$ \\
\hline *
\end{tabular}

* medianas seguidas de mesma letra não diferem estatisticamente
As análises ao microscópio eletrônico de varredura, realizadas nas secções longitudinais dos espécimes, revelaram três diferentes regiões: a) metal; b) zona de interação metal/cerâmica; c) cerâmica. Foi verificado que todas as interfaces apresentaram-se intactas, com ótimo contato e molhabilidade entre a cerâmica e a liga de Co-Cr, assim como ausência de falhas ou fendas, sugerindo adequada adesão entre os dois materiais, conforme demonstrado nas Figuras 4, 5 e 6.

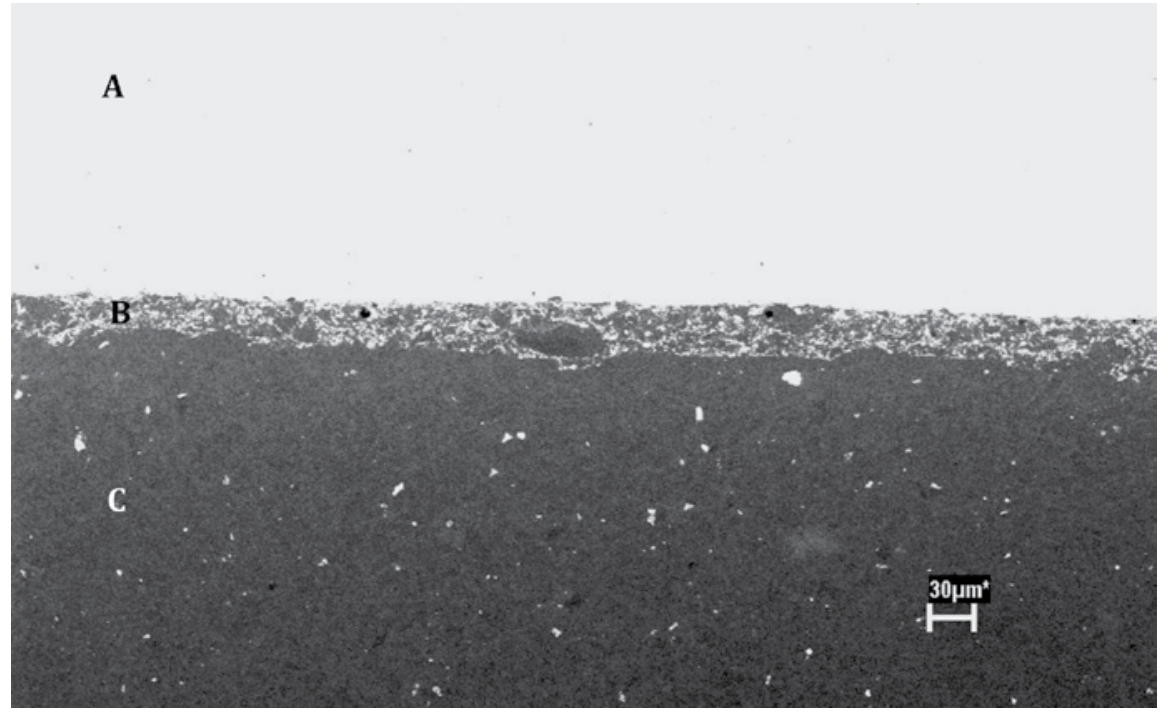

Figura 4 - Microfotografia representativa do MEV (150x) da cerâmica Vita Omega 900 - superfície da liga de $\mathrm{Co}-\mathrm{Cr}$, com a queima da camada de opaco a $900^{\circ} \mathrm{C}$. Notar boa união entre a cerâmica e metal a) metal, b)zona de interação, c) cerâmica. 


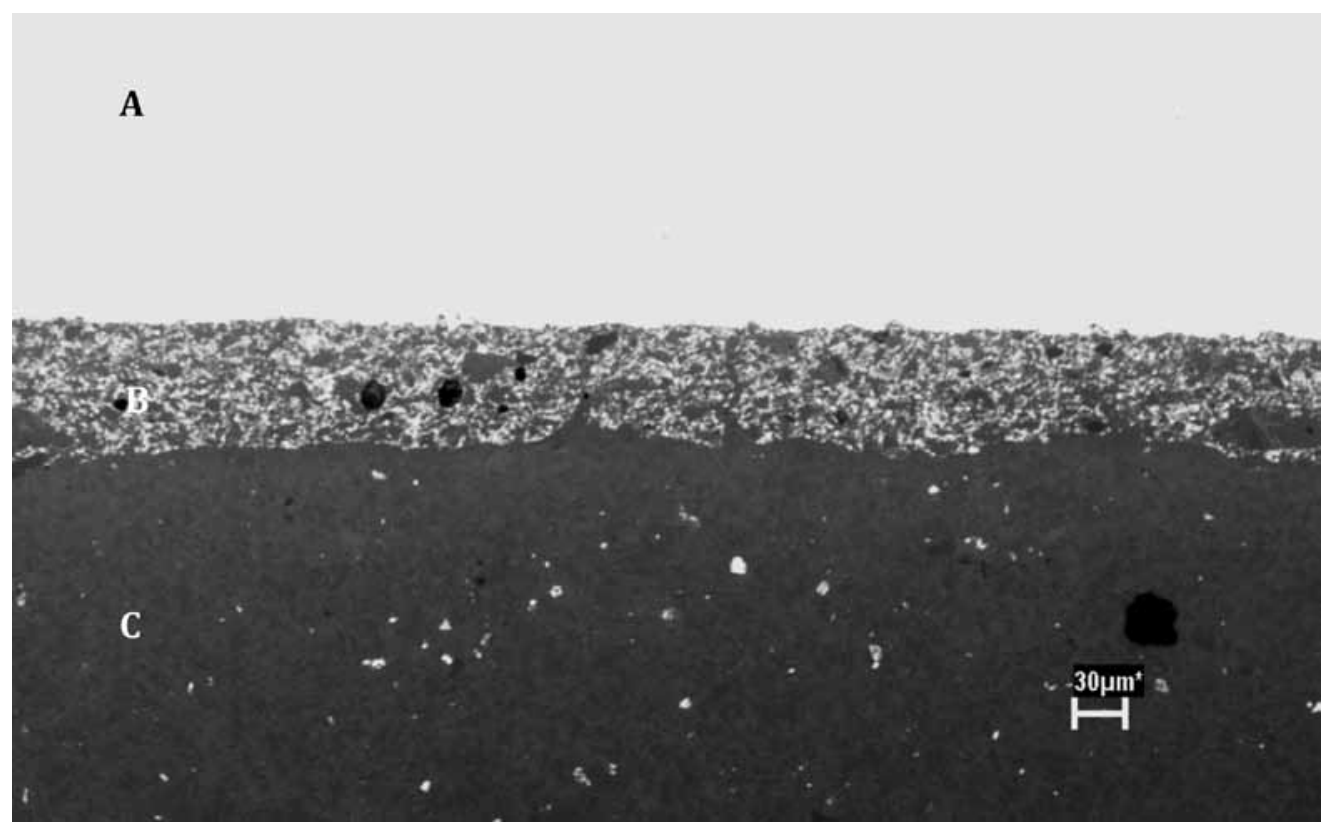

Figura 5 - Microfotografia representativa do MEV (150x) da cerâmica Vita Omega 900 - superfície da liga de Co-Cr, com a queima da camada de opaco a $950^{\circ} \mathrm{C}$. Notar boa união entre a cerâmica e metal a) metal, b) zona de interação, c) cerâmica.

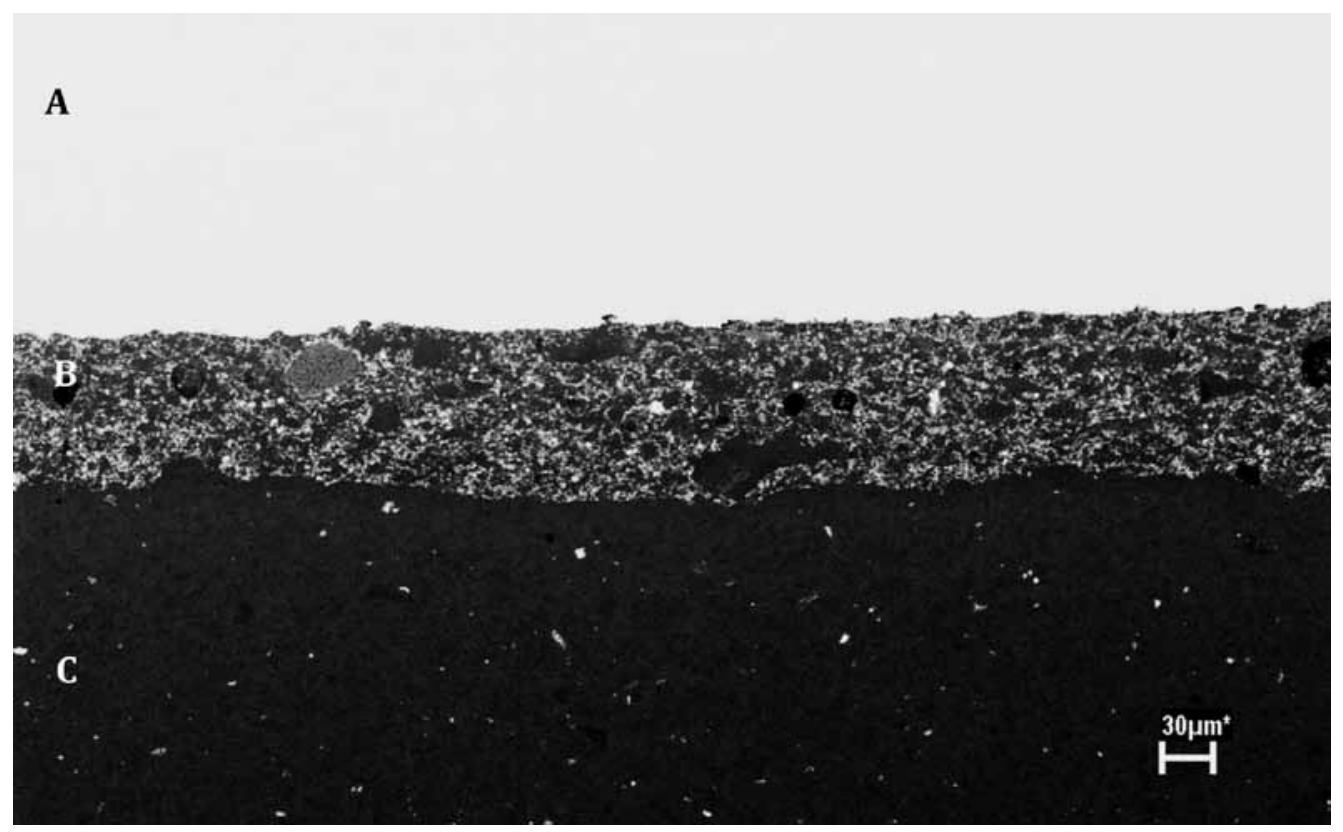

Figura 6 - Microfotografia representativa do MEV (150x) da cerâmica Vita Omega 900 - superfície da liga de Co-Cr, com a queima da camada de opaco a $1000^{\circ} \mathrm{C}$. Notar boa união entre a cerâmica e metal a) metal, b) zona de interação, c) cerâmica.

Nas análises em estereomicroscópio, com aumento de 30x, o grupo $1\left(900^{\circ} \mathrm{C}\right)$ apresentou falhas adesivas na zona interfacial camada de opaco/Co-Cr, sem a presença de cerâmica na superfície do metal, porém também foi observado uma escura camada de óxido em todos os espécimes. Enquanto que nos outros grupos $\left(950\right.$ e $\left.1000^{\circ} \mathrm{C}\right)$, foi verificada em todos os espécimes, a presença de cerâmica e por vezes cerâmica de corpo na superfície do metal, sugerindo falhas coesivas.

As imagens representativas da superfície do metal e da cerâmica após o teste de flexão de três pontos de todos os grupos estão ilustradas nas Figuras 7 a-c. A hipótese foi aceita. 

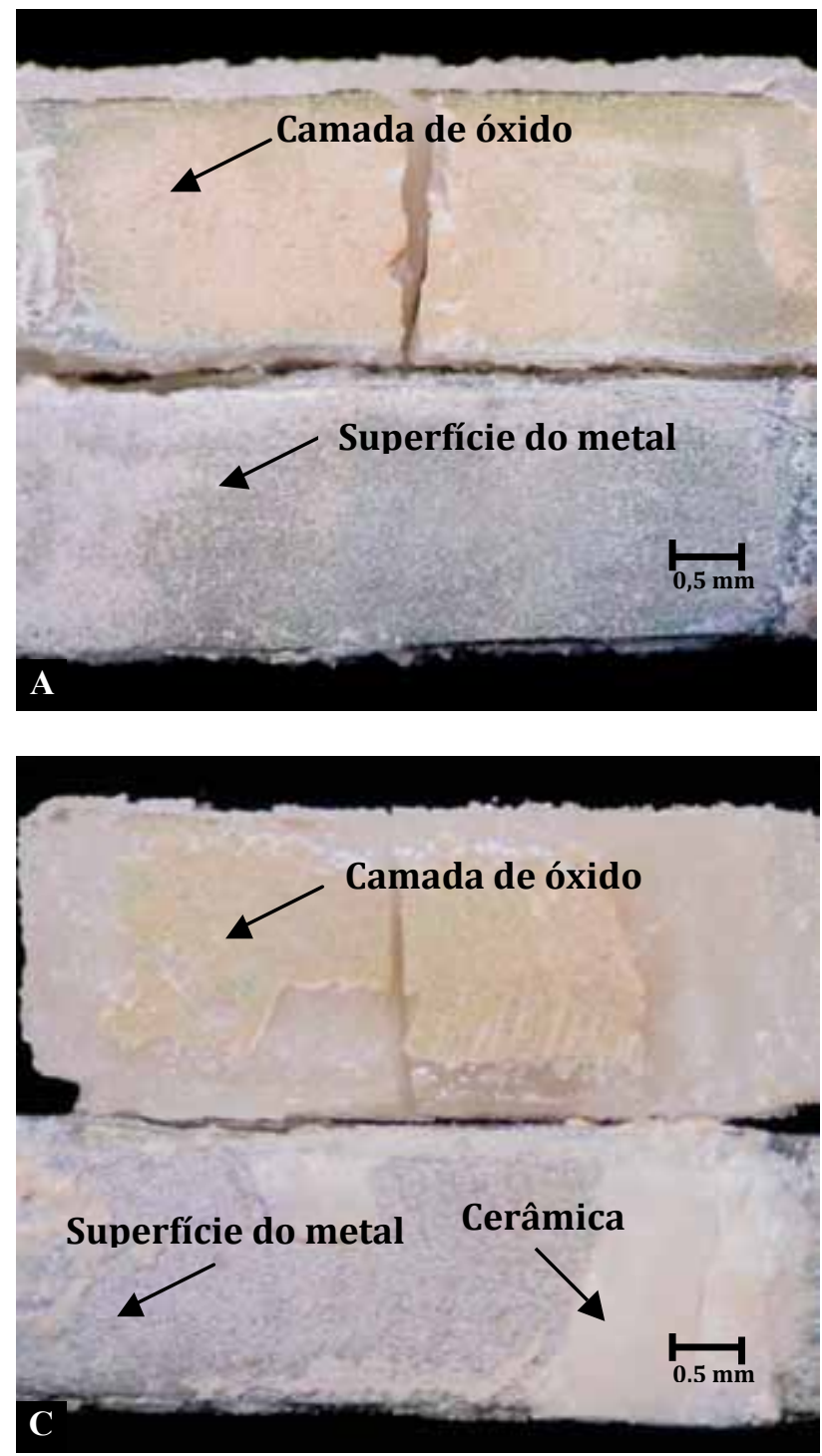

\section{DIscussão}

Neste estudo avaliou-se a resistência de união entre a liga odontológica de cobalto-cromo (Wirobond ${ }^{\circledR}$ C, Bego) e a cerâmica feldspática (Vita Omega 900, Vita Zahnfabrick) devido à viabilidade de utilização da liga de cobalto-cromo para confecção de IE de prótese parciais fixas e unitárias e aos poucos estudos relatados na literatura sobre este assunto.

Vários testes in vitro têm sido propostos para mensurar a resistência de união entre o metal e a cerâmica incluindo os testes de tração $0^{6,25}$, cisalhamento $^{5,15,16,24}$, flexão de três pontos ${ }^{2,12,20,27,28}$ e flexão de quatro pon$\operatorname{tos}^{7,10}$, uma vez que na literatura não há um consenso em relação ao teste mais indicado para a avaliação da resistência de união entre esses dois materiais. Por

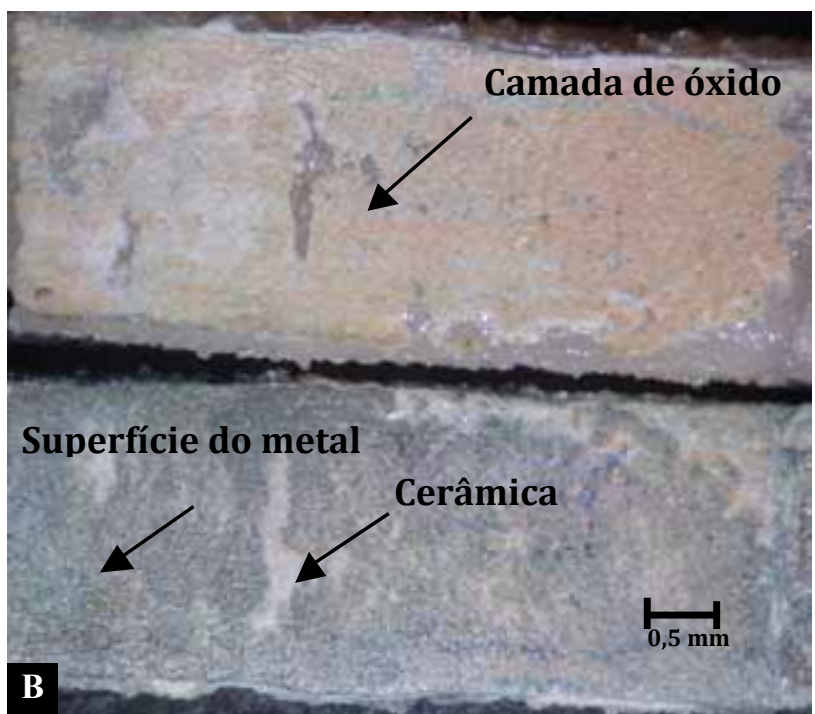

Figura 7a-c - Imagens obtidas por meio da lupa estereomicroscópica dos espécimes Vita Omega 900/Co-Cr (30x), após o teste de flexão a) ausência de fragmentos de cerâmica e camada de óxido na superfície de Co-Cr no G1 $\left.\left(900^{\circ} \mathrm{C}\right), \mathrm{b}\right)$ fragmentos da camada de opaco na superfície de Co-cr nos G2 e c) G3.

outro lado, Della Bona e van Noort ${ }^{11}$, analisando o ensaio de cisalhamento, observaram que este tipo de teste gera em todas as amostras fraturas coesivas em forma de arco. Isto ocorre devido à distribuição de tensão altamente não uniforme na interface dos materiais. Já o teste de resistência à flexão, é o teste que mais simula as condições clínicas, já que no momento do ensaio as amostras sofrem forças de compressão, tração e cisalhamento simultaneamente ${ }^{1}$. Além disso, o teste de flexão de três pontos é o teste recomendado pela International Organization for Standardization ${ }^{18}$ para análise da resistência de união metal/cerâmica. Por esses motivos, o teste de resistência à flexão de 3 pontos foi o ensaio selecionado para este estudo, o qual demonstrou ser efetivo para avaliar a resistência de união entre o metal e cerâmica utilizando três diferentes temperaturas de queima da camada de opaco. 
No presente estudo foram utilizadas três diferentes temperaturas de queima da camada de opaco, $900^{\circ} \mathrm{C}$ recomendada pela fabricante, e duas experimentais $950^{\circ} \mathrm{C}$ e $1000^{\circ} \mathrm{C}$. Os resultados de resistência flexural observados nos grupos em que a temperatura foi maior do que aquela recomendada pelo fabricante, ou seja, com temperatura de $950^{\circ} \mathrm{C}(\mathrm{Gr} 2=19,84 \pm 4,24 \mathrm{~N})$ e $1000^{\circ} \mathrm{C}(\mathrm{Gr} 3=22,43 \pm 5,94 \mathrm{~N})$ foram estatisticamente superiores aos resultados observados no grupo com temperatura de $900^{\circ} \mathrm{C}$. A técnica de elevação desta temperatura é baseada na hipótese de que há um aumento na transferência de elétrons entre o vidro e os óxidos do $\mathrm{metal}^{8}$, sugerindo o aumento da resistência de união entre o metal e a cerâmica. Wight et al. ${ }^{26} \mathrm{e} \mathrm{Hammad} \mathrm{et}$ al. ${ }^{17}$ avaliaram o efeito do aumento da temperatura de queima da camada de opaco, utilizando temperaturas de $26^{\circ} \mathrm{C}$ e $18^{\circ} \mathrm{C}$ acima daquela recomendada pelo fabricante, respectivamente. Os autores concluíram que a elevação da temperatura melhorou a resistência de união entre o metal e a cerâmica, vindo a corroborar com este estudo. Contudo, os resultados do presente estudo revelaram que todos os grupos apresentaram valores de resistência flexural maiores que o mínimo recomendado, $5,625 \mathrm{~N}$, estabelecido pela DIN $13.927^{14}$.

A situação de maior resistência entre metais básicos e cerâmica é demonstrada quando a fratura ocorre no interior da cerâmica e não na interface ${ }^{9,26}$. No presente estudo, após a avaliação em lupa estereoscópica dos espécimes testados, nos grupos experimentais de $950^{\circ} \mathrm{C}$ e $1000^{\circ} \mathrm{C}$, verificou-se sobre a superfície do metal a presença da camada de opaco e de cerâmica de corpo e sobre a superfície da cerâmica notou-se interrupções da camada de óxido. Por outro lado, no grupo de $900^{\circ} \mathrm{C}$ foi observada camada de óxido ao longo de toda superfície da cerâmica e verificou-se ausência da camada de opaco e de cerâmica sobre a superfície do metal (Figura 7a-c). Estes resultados estão de acordo com os trabalhos de Wight et al. ${ }^{26} \mathrm{e}$ Hammad et al. ${ }^{17}$, nos quais houve melhora da união entre metal e cerâmica com o aumento da temperatura de queima da camada de opaco, sendo observadas fraturas dentro da cerâmica para as amostras queimadas em temperaturas maiores que a recomendada pelo fabricante, enquanto que as amostras queimadas em temperatura recomendada pelo fabricante apresentaram fraturas exclusivamente na interface.

As imagens obtidas em estereomicroscópio sugerem que com o aumento da temperatura de queima da camada de opaco, foi possível melhorar a resistência de união entre a camada de opaco e a liga de Co-Cr.

Os resultados obtidos no presente estudo podem não refletir precisamente a situação in vivo, como por exemplo, a maneira da aplicação da carga. Neste estudo a resistência à flexão foi determinada, aplicando a carga até a ruptura, em ponto único; enquanto que, nas condições in vivo as falhas ocorrem sob cargas pequenas e moderadas, aplicadas repetidamente por um longo período. Visando simular melhor as condições encontradas no meio bucal, avaliações após ciclagem térmica e mecânica deveriam ser executadas posteriormente para avaliar a união entre metal e cerâmica nas diferentes temperaturas de queima de opaco.

\section{Conclusão}

Dentro das limitações deste estudo, pode-se concluir que a elevação da temperatura da camada de queima de opaco influenciou positivamente, de maneira significante, a resistência de união entre o metal e a cerâmica.

\begin{abstract}
This study evaluated the opaque layer firing temperature on the flexural strength of a ceramic fused to commercial cobaltchromium alloy $(\mathrm{Co}-\mathrm{Cr})$. The hypotheses were that opaque layer temperature increasing get higher the metal/ceramic bond strength. Metallic frameworks $(25 \times 3 \times 0.5 \mathrm{~mm})(\mathrm{n}=30)$ were cast in $\mathrm{Co}-\mathrm{Cr}$ and airborne particle abraded with 150 $\mu \mathrm{m}$ aluminum oxide at the central area of the frameworks $(8 \times 3 \mathrm{~mm})$ and after divided into three groups, according to opaque layer firing temperature ( $\mathrm{n}=10)$ : $\mathrm{G} 1$ (control) $-900^{\circ} \mathrm{C} ; \mathrm{G} 2-950^{\circ} \mathrm{C}$ and $\mathrm{G} 3-1000^{\circ} \mathrm{C}$. Opaque ceramic were applied and the glass ceramic (Vita Omega 900, Vita Zahnfabrick, Bad Säckingen, Germany) was fired onto them (thickness: $1 \mathrm{~mm}$ ). The specimens were kept in distilled water at $37^{\circ} \mathrm{C}$ for 24 hours, after that it were submitted to three points flexural strenght test. After the test, failure types were noted. The data were analyzed using 1 way Kruskal-Wallis and Dunn test $(5 \%)$. The difference in mean flexure strength values were statistically significant $(\mathrm{p}=0,004)$. The groups G2$19,84 \pm 4,24 \mathrm{~N}$ and G3- 22,43 $\pm 5,94 \mathrm{~N}$ presented higher flexure strength values than G1- $12,525 \pm 1,08 \mathrm{~N}$. It was concluded that the opaque layer temperature increasing improved the flexure bond strength values significantly. The failure type predominant was adhesive at metal/ceramic interface for group G1 sample and exclusively cohesive at the opaque ceramic/Co-Cr interfacial zone with presence of ceramic on the metal surface for groups G2 and G3.
\end{abstract}

\title{
UNITERMS
}

Dental materials; metal ceramic alloys; ceramic; chromium alloy. 


\section{REFERÊNCIAS}

1. Anusavice KJ. Phillips Materiais dentários. 11 ed. Rio de Janeiro: Elsevier, 2005. 763p.

2. Atsü S, Berksun S. Bond strength of three porcelains to two forms of titanium using firing atmospheres. J Prosthet Dent. 2000;84(5):567-74.

3. Baran, GR. Selection criteria for base metal alloys for use with porcelans. Dent Clin North Am. 1985;29(4):779-87

4. Barghi N, McKeehan-Whitmer, Aranda R. Comparison of fracture strenght of porcelain-veneered-to-high noble and base metal alloy. J Prosthet Dent. 1987;57(1):23-6.

5. Bondioli IR, Bottino MA. Evaluation of shear bond strebgth at the interface of two porcelains and pure titanium injected into the casting mold at three different temperatures. J Prosthet Dent. 2004;91(6):541-7.

6. Bullard JT, Dill RE, Marker VA, Payne EV. Effects of sputtered metal oxide films on the ceramic-to-metal bond. J Prosthet Dent. 1985;54(6);776-8.

7. Caputo AA. Effect of surface preparation on bond strenght of nonprecious e semi-precious alloys. J Calif Dent Assoc. 1979;6(11):42-6.

8. Daftary F, Donovan T. Effect of electrodeposition of gold on porcelainto-metal bond strength. J Prosthet Dent. 1987;57(1):41-6.

9. Deger S, Caniklioglu MU. Effects of tin plating on base metal alloyceramic bond strength. Int J Prosthodont. 1998;11(2):165-72.

10. DeHoff PH, Anusavice KJ, Hathcok PW. An evaluation of the fourpoint flexural test for metal-ceramic bond strenght. J Dent Res. 1982;61(9):1066-9.

11. Della Bona A, Van Noort R. Shear vs. Tensile Bond Strength of Resin Composite Bonded to Ceramic. J Dent Res. 1995;74(9):1591-6.

12. Galindo DF, Ercoli C, Graser GN, Tallents RH, Moss ME. Effect of soldering on metal-porcelain bond strenght in repaired porcelain-fusedto-metal castings. J Prosthet Dent. 2001; 85(1):88-94.

13. Gavelis JR, Lim SB, Guckes AD, Morency JD, Sozio RB. A comparison of the bond strength of two ceramometal systems. J Prosthet Dent. 1982;48(4):424-8.

14. German Standard Institution, DIN 13.927 metal-ceramic system. Berlin: Beuth; 1990.

15. Graham JD, Johnson A, Wildgoose DG, Shareef MY, Cannavina G. The effect of surface treatments on the bond strength of a nonprecious alloys - ceramic interface. Int J Prosthodont. 1999;12(4):330-4.

16. Guinn JW, Griswold WH, Vermilyea SG. The effect of cooling rate on the apparent bond strenght of porcelain-metal couples. J Prosthet Dent. 1982;48(5):551-4.

17. Hammad IA, Goodkind RJ, Gerberich WW. A shear test for yhe bond strenght of ceramometals. J Prosthet Dent. 1987;58(4):431-7.
18. International Organization of Standardization. Metal-ceramic dental restorative systems: Standard n 9693. Geneva, 1999, p. 1-14.

19. Kelly JR, Rose TC. Nonorecious alloys for use in fixed prosthodontics: a literature review. J Prosthet Dent. 1983;49(3):363-70

20. Lavigne MH, Custer F. Variables affecting the strength of bond between porcelain and gold. J Dent Res. 1966;45(1):32-6.

21. MacEntee MI, Hawbolt EB, Zahel JI. The tensile and shear strength of a base metal weld joint used in dentistry. J Dent Res. 1981;60(2):154-8.

22. Maclean JW. The metal-ceramic restoration. Dent Clin North Am. 1985;27(4):747-61.

23. Okazaki M, Wang X, Toguchi MS, Taira M, Takahashi J, Matsuo C, et al. Improvement of bond strength in metal-ceramic systems using a gold intermediate layer. Dent Mater J. 1998;17(3):163-73.

24. Stannard JG, Marks L, Kanchanatawewant K. Effect of multiple firing on the bond strength of selected matched porcelai-fused-to-metal combinations. J Prosthet Dent. 1990;63(6):627-9.

25. Uusalo EK, Lassila VP, Yki-Urpo AU. Bonding of dental porcelain to ceramic-metal alloys. J Prosthet Dent. 1987;57(1):26-9.

26. Wight TA, Bauman JC, Pelleu Junior GB. An evaluaton of four variables affecting the bond strength of porcelain to nonprecious alloy. $\mathrm{J}$ Prosthet Dent. 1977;37(5):570-7.

27. Wood MC, Thompson GA, Agar JR. A comparison of debonding strenghts of four metal-ceramic systems with and without opaque porcelain. J Prosthet Dent. 2007;97(3):141-9.

28. Wu Y, Moser JB, Jameson LM, Malone WF. The effect of oxidation heat treatment on porcelain bond strenght in sekected base metal alloys. J Prosthet Dent. 1991;66(4);439-44.

Recebido em 28/09/2007 Aprovado em 07/04/2010

Correspondência: Luis Gustavo Oliveira de Vasconcellos Endereço: Alameda Harvey C. Weeks, 14 sala 09 - Vista Verde São José dos Campos - São Paulo -Brasil CEP: $12223-830$ e-mail: lgovasconcellos11@terra.com.br 